\title{
Anterior Transverse Approach in Osteosynthesis of a Type II Fracture of the Coronoid Process in an Adolescent
}

\author{
Mohamed Rida El Galiou ${ }^{*}$, E.Y. Houass, M. Boufettal, R.A. Bassir, M. Kharmaz, M.O. Lamrani, M.S. Berrada
}

\author{
Department of Orthopedics and Traumatology, University Hospital Center of Rabat, Faculty of Medicine and Pharmacy of Rabat, University \\ Mohammed V Souissi, Rabat, 10000, Morocco
}

DOI: $10.36347 /$ sjams.2020.v08i12.012

| Received: 24.11.2020 | Accepted: 05.12.2020 | Published: 14.12.2020

*Corresponding author: Mohamed Rida El Galiou

Abstract

The anterior transverse elbow approach for the management of coronoid process fractures is not common. The authors report the case of an 18-year-old youngster presenting with a type II coronoid fracture according to Regan and Morrey, treated surgically, by anterior approach, with compressive osteosynthesis by two mini screws. At a follow-up of six months, the clinical radiological evolution was satisfactory.

Keywords: Coronoid process, anterior approach, dislocation fracture.

Copyright $(\odot 2020$ The Author(s): This is an open-access article distributed under the terms of the Creative Commons Attribution 4.0 International License (CC BY-NC 4.0) which permits unrestricted use, distribution, and reproduction in any medium for non-commercial use provided the original author and source are credited.

\section{INTRODUCTION}

Isolated fractures of the coronoid process are not frequent. On the other hand, they are frequently associated with posterior dislocations of the elbow, thus compromising its stability. When internal fixation of the coronoid process is necessary, direct control of the fracture site simplifies reduction and synthesis, compared to recall screwing. Direct screwing also gives the lesion better stability.

\section{Clinical Observation}

This is an 18-year-old male, left-handed adolescent, presenting to the emergency room for treatment of a trauma to the left elbow following a sports accident with landing on the palm of the hand. Clinical examination found an upper limb trauma attitude, pain, diffuse edema of the elbow and complete functional impotence of the limb. The vasculo-nervous examination was unremarkable. The elbow x-ray revealed a postero-external dislocation fracture of the elbow (fig. 1). The patient benefited at $\mathrm{H} 4$ from a reduction in his dislocation under general anesthesia. Post-reduction examination revealed instability in the elbow. The follow-up radiograph showed the reduction in dislocation associated with a displaced fracture of the coronoid process classified as type II according to Regan and Morrey (Fig. 2). The decision was to go for surgical treatment. Under local anesthesia and by transverse anterointernal route of the elbow (fig. 3), along the internal edge of the biceps muscle without exceeding the elbow fold, the brachial vasculo-nervous bundle is not dissected, an anatomical passage is offered between the biceps muscle and the vasculo-nervous bundle, the brachialis muscle is discarded in the direction of its fibers at the level of the $2 / 3$ lateral $1 / 3$ medial junction, up to the joint capsule incised longitudinally directly on the coronoid process, then we proceeded to the reduction of the displaced fragment and its compression osteosynthesis by two $1.5 \mathrm{~mm}$ mini screws (fig. 4). The left upper limb was immobilized by a posterior brachioantébrachial splint for fifteen days. The postoperative consequences were simple. Rehabilitation was authorized after 15 days. Consolidation was obtained on day 45 . With a sixmonth follow-up, the mobility of the elbow was normal.
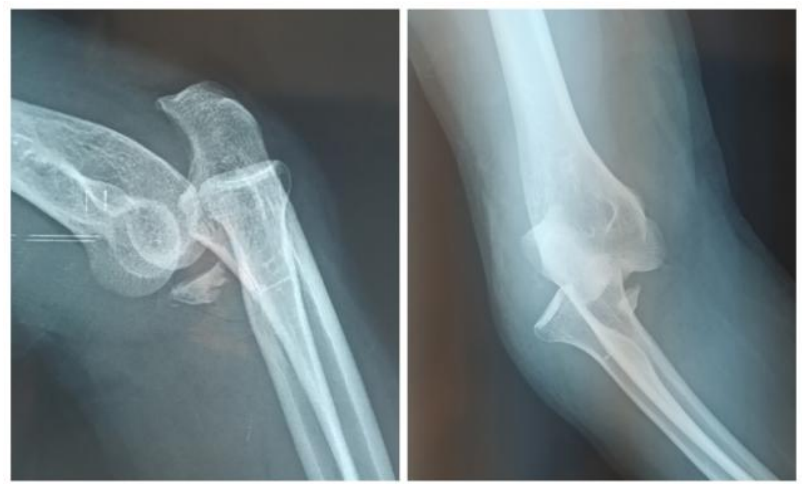

Fig-1:X-ray of the elbow, face and profile, showing a postero-external dislocation fracture of the elbow 


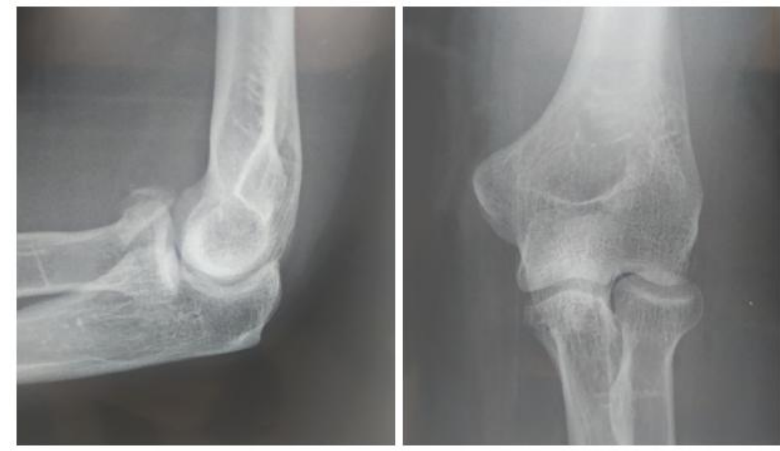

Fig-2: Control X-ray of the elbow showing reduction of the dislocation with a fracture of the coronoid process

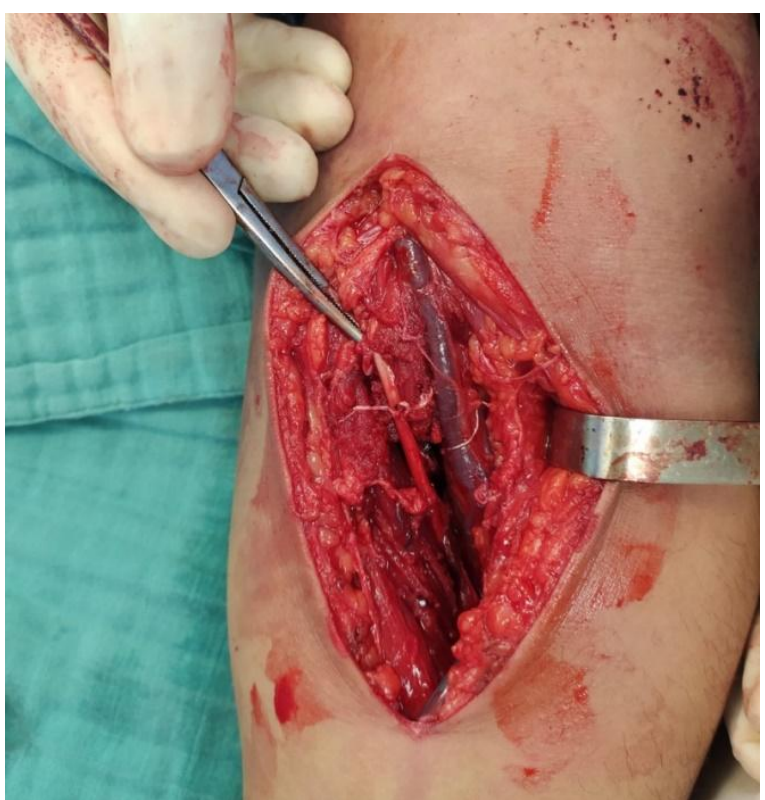

Fig-3: Intraoperative view showing the brachial vascular bundle and the median nerve

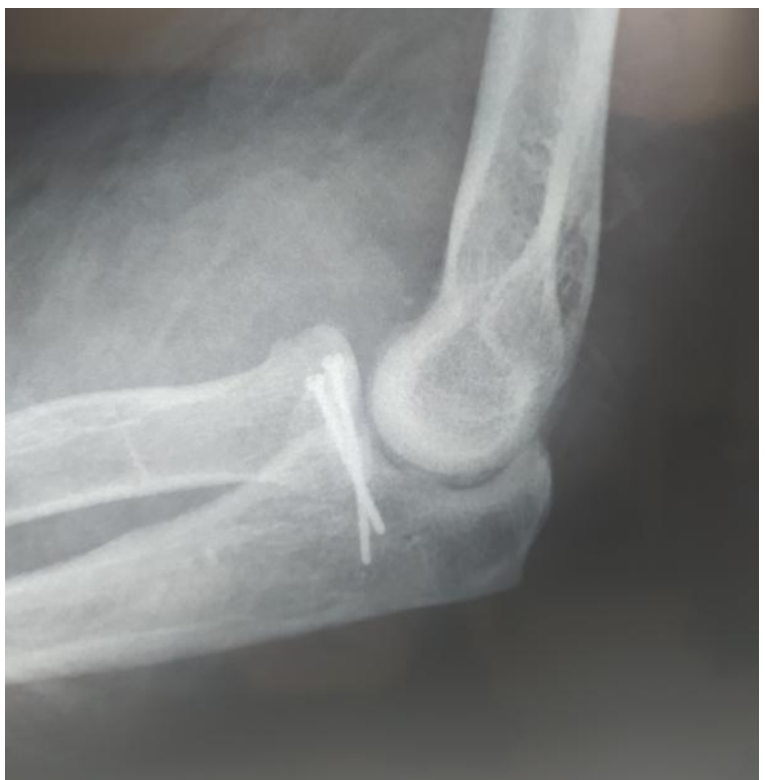

Fig-4: Control x-ray showing osteosynthesis by two miniscrews

\section{DISCUSSION}

Fracture of the coronoid process is rarely isolated [1]. It is generally part of a dislocated elbow trauma, and is almost always associated with radial head fracture and / or medial collateral ligament injury.

Regan and Morrey [1] classified coronoid fractures into 3 types according to the level of injury: in type 1 , only the tip of the coronoid is fractured; in type 2 , half the volume of the coronoid is fractured along a line parallel to the major axis of the ulna and in type 3 which corresponds to a fracture of the coronoid base.

In the case of a dislocated elbow fracture, the preoperative or especially post-reduction radiographic assessment requires the systematic search for the fracture of the coronoid and the assessment of its displacement and size.

Orthopedic treatment is reserved for types I and II with complete post-reduction stability. The results of orthopedic treatment are so far good or very good for fractures taking less than $25 \%$ of the height of the process. Surgical treatment is indicated in the event of type II fracture with instability of the elbow and type III. According to Doornberg [2], patients with fractures greater than $25 \%$ of the heights of the process who have benefited from surgical treatment have mostly excellent results. The functional result seems to be related to the size of the fracture fragment. Surgical treatment recommends the use of the anterior approach rather than the posterior approach, which does not offer control over the fracture fragment and only allows screwdriving with less stability, unlike the anterior approach [3]. Ameur [4] proposed a more direct approach through the tendon of the brachialis. Compression screwing is facilitated by the use of cannulated screws with distal threads. In this case, a guide wire is used instead of the drill bit. The screw is then placed on the guide wire while controlling its length through the endo-articular route. The temporary reduction spindle which allowed rotation control during tightening can then be removed. On the other hand, Ring [5] describes screwing as an unsuitable treatment, the reference according to him being the fixation by buttress plate. Functional results are assessed according to the functional score of the Mayo Elbow Performance Index (MEPI), studying pain, mobility, stability and function [3].

\section{CONCLUSION}

The surgical treatment of fractures of the coronoid process by an anterior approach by longitudinal dissociation of the brachialis muscle, allows direct, more stable screwing, with a rapid and satisfactory functional recovery. 


\section{REFERENCE}

1. Regan W, Morrey B. Fractures of the coronoid process of the ulna. J Bone Joint Surg Am. 1989; 71: 1348-1354.

2. Doornberg J, Ring D. Coronoid fracture patterns. J Hand Surg. 2006; 31A (1): 45-52.

3. Belmoubarik A, Ahed K, Abouchane M, Mahraoui MA, Elandaloussi Y, Haddoun AR, Nechad M. Voie antérieure transversale dans l'ostéosynthèse d'une fracture type III du processus coronoïde chez un adolescent: à propos d'un cas et revue de literature. Pan African Medical Journal. 2015;20(1).

4. Ameur NE, Rebouh M, Oberlin C. Anterior transbrachial approach of the coronoid apophysis. Chir Main. 1999; 18: 220-225.

5. Ring D, Jupiter JB, Zilberfarb J. Posterior dislocation of the elbow with fractures of the radial head and coronoid. J Bone Joint Surg Am. 2002 Apr; 84-A(4):547-51. 